submitted to Astrophysical Journal Letters

Preprint typeset using IATEX style emulateapj v. 11/12/01

\title{
THE EPOCH OF REIONIZATION IN MODELS WITH REDUCED SMALL SCALE POWER
}

\author{
Rachel S. Somerville ${ }^{1}$, James S. Bullock ${ }^{2,3}$, \& Mario Livio ${ }^{1}$
}

submitted to Astrophysical Journal Letters

\begin{abstract}
Reducing the power on small scales relative to the 'standard' $\Lambda$ CDM model alleviates a number of possible discrepancies with observations, and is favored by the recent analysis of WMAP plus galaxy and Lyman- $\alpha$ forest data. Here, we investigate the epoch of reionization in several models normalized to $W M A P$ on large scales, and with sufficiently reduced power on small scales to solve the halo concentration and substructure problems. These include a tilted model, the WMAP running-index model, and a Warm Dark Matter model. We assume that the Universe was reionized by stellar sources comprised of a combination of supermassive $\left(\sim 200 M_{\odot}\right)$ Pop III stars and Pop II stars with a 'normal' IMF. We find that in all of these models, structure formation and hence reionization occurs late, certainly at redshifts below ten, and more probably at $z \lesssim 6$. This is inconsistent (at $2 \sigma$ ) with the determination of $z_{\text {reion }} \simeq 17$ from the $W M A P$ temperature-polarization data and is only marginally consistent with SDSS quasar observations. The tension between the galactic-scale observations, which favor low-power models, and the early reionization favored by WMAP can only be resolved if the efficiency of Pop III star formation is dramatically higher than any current estimate, or if there is an exotic population of ionizing sources such as mini-quasars. Otherwise, we may have to live with the standard $\Lambda$ CDM power spectrum, and solve the small-scale problems in some other way.
\end{abstract}

Subject headings: cosmology: theory — galaxies: evolution — intergalactic medium

\section{INTRODUCTION}

The first-year WMAP satellite data have yielded a remarkable confirmation of what is coming to be regarded as the 'Standard Model' of cosmology: a Hubble parameter $H_{0} \simeq 70 \mathrm{~km} \mathrm{~s}^{-1} \mathrm{Mpc}^{-1}$, matter and vacuum densities consistent with a flat geometry $\Omega_{m} \simeq 1-\Omega_{\Lambda} \simeq 0.3$, and a nearly scale-invariant primordial power spectrum, $n_{s} \simeq 1$, at least on large scales. There were some surprises, as we will discuss below, but in many ways the WMAP results highlight the marked success of standard $\Lambda \mathrm{CDM}$ in reconciling diverse observations from the $\mathrm{CMB}$, to galaxy clusters, supernovae, and gravitational lensing. This remarkable agreement with data on large scales, however, brings into sharper focus several nagging discrepancies on small-scales. Specifically, observed galaxy central densities appear to be significantly lower than the standard $\Lambda$ CDM model predicts (Flores \& Primack 1994; Moore 1994; Alam et al. 2002; Swaters et al. 2003; McGaugh et al. 2003; van den Bosch et al. 2003) and $\Lambda$ CDM may also produce too much substructure compared with observed numbers of satellite galaxies in the Local Group (Klypin et al. 1999; Moore et al. 1999). A natural way to relieve these problems, while maintaining the large-scale success of the model, is to reduce the power on small scales relative to large, either by introducing a 'tilt' in the primordial power spectrum $\left(n_{s}<1\right)$, as expected in some variants of inflation (Alam et al. 2002; Zentner \& Bullock 2002), or by resorting to Warm Dark Matter (WDM) (Avila-Reese et al. 2001; Bode et al. 2001; Alam et al. 2002). In these models, collapse happens later, when the Universe was less dense, and galaxy halos are naturally less centrally concentrated. Later collapse also alleviates the dwarf problem
(Colín et al. 2000; Knebe et al. 2002; Bullock \& Zentner 2002; Zentner \& Bullock 2003), and the 'angular momentum catastrophe' - the ongoing problem with producing disk galaxies with sufficient specific angular momentum in hydrodynamic simulations within the $\Lambda \mathrm{CDM}$ framework (Sommer-Larsen \& Dolgov 2001). Interestingly, one of the surprises of the WMAP analysis is that it favors (at $\sim 2 \sigma$ ) a model in which the spectral index varies strongly as a function of wavenumber, $\mathrm{d} n / \mathrm{d} \ln k \simeq-0.03$ (Spergel et al. 2003), in precisely the manner needed to cure many of the problems on small-scales (Zentner \& Bullock 2003).

The second (and perhaps major) surprise from the $W M A P$ report was the detection of a large amplitude signal in the TE maps, indicating a large optical depth to Thomson scattering $(\tau=0.17 \pm 0.04)$. The straightforward interpretation of this result is that the Universe became reionized at $z_{\text {reion }}=17 \pm 5$ (Kogut et al. 2003), rather earlier than many had expected. Several workers have recently demonstrated that even within the standard $\Lambda$ CDM framework, models of reionization require rather extreme assumptions in order to produce enough early star or quasar formation to reionize the Universe by $z \sim 17$ (Wyithe \& Loeb 2003; Haiman \& Holder 2003; Ciardi et al. 2003a; Sokasian et al. 2003; Cen 2003). Put in the context of the small-scale crises facing the standard model, and indeed, in view of the running-index model favored by the WMAP analysis, we are left with a paradox. The galactic-scale data favor low-power models precisely because they produce late structure formation, but the high optical-depth measurement seems instead to favor early collapse. In fact, the late reionization implied by low-power models would more easily accommodate the

${ }^{1}$ Space Telescope Science Institute, Baltimore MD 21218; somerville@stsci.edu; mlivio@stsci.edu

2 Harvard-Smithsonian Center for Astrophysics, Cambridge, MA 02138; jbullock@cfa.harvard.edu

3 Hubble Fellow 
simplest interpretation of the Gunn-Peterson troughs observed in several SDSS quasars at $z \gtrsim 6$ (Fan et al. 2001; Becker et al. 2001), and the high temperature of the IGM at $z \sim 4$ (Hui \& Haiman 2003).

In this Letter we set out to quantify these concerns using a simple yet conservative model that is informed and motivated by numerical simulations. Our aim is to compute conservative upper limits on $z_{\text {reion }}$ for several models that have small-scale power reduced to plausibly solve the galactic-scale difficulties, including the running spectral index model favored by WMAP. In what follows, we assume that reionization is due to stellar sources alone, as AGN are likely to be unimportant to reionization at high redshift unless there are exotic objects (such as 'miniquasars') that is completely disjoint from the known population (Schirber \& Bullock 2003).

\section{MODELS AND NORMALIZATION}

In order to focus on the ramifications of reducing the small-scale power, we will fix the cosmological parameters in all of our models to canonical values: $\Omega_{m}=1.0-\Omega_{\Lambda}=$ $0.3, \Omega_{b} h^{2}=0.02$, and $H_{0}=70 \mathrm{~km} \mathrm{~s}^{-1} \mathrm{Mpc}^{-1}$. Additional parameters of each of the models are described in Table 1. In all cases, we assume that the primordial power spectrum takes the form $P(k) \propto k^{n_{s}}$, with a spectral index that can vary as function of scale as: $n_{s}=n_{s}\left(k_{0}\right)+0.5 \mathrm{~d} n / \mathrm{d} \ln k \ln \left(k / k_{0}\right)$ (e.g. Kosowsky \& Turner 1995; Hannestad et al. 2002). In the second column of Table 1 , we list $n_{s}$, evaluated at $k_{0}=k_{W M A P} \equiv 0.05$ $\mathrm{Mpc}^{-1}$ and in column 3, we give $\mathrm{d} n / \mathrm{d} \ln k$. In order to make contact with previous work, our specific choice for normalizing standard $\Lambda \mathrm{CDM}$ (with $n_{s}=1.0$ ) is $\sigma_{8}=0.9$, where $\sigma_{8}$ (column 4) is the linear, rms fluctuation amplitude of the power spectrum within spheres of radius $8 \mathrm{~h}^{-1} \mathrm{Mpc}$, evaluated at $z=0$. This spectral index and normalization are very close to the best-fit parameters derived from the WMAP data alone, without combination with other data sets. In order to ensure consistency on large scales, we have chosen to fix the normalization of the rest of our models to match that of standard $\Lambda \mathrm{CDM}$ at $k=k_{W M A P}$.

The first of our models with reduced small-scale fluctuations, "TILT", has $\sigma_{8}=0.75$ and modest tilt, $n_{s}=0.95$, as expected in many simple models of inflation (see, e.g. Kinney 2003). The specific parameter choice is motivated by the work of Zentner \& Bullock $(2002 ; 2003)$ who concluded that this normalization/tilt is favored by galaxy rotation curve data, and may also alleviate the dwarf satellite problem without the need for differential feedback. Interestingly, this parameter choice is also nearly identical to the best-fit power-law model in the WMAP joint analysis with other CMB data, the 2dF Galaxy Redshift Survey, and Lyman $\alpha$ forest data (Spergel et al. 2003). The next model, "RSI", is motivated by the same joint WMAP analysis, but represents the case in which they have allowed a Running Spectral Index. Their best fit has $n_{s}=0.93$ and $\mathrm{d} n / \mathrm{d} \ln k=-0.03$. Our normalization for RSI, $\sigma_{8}=0.81$, is slightly below the quoted WMAP value (by $\sim 3.5 \%$ ) because of the simple way we have elected to normalize on large scales, but this difference is small compared to the quoted uncertainty. This RSI case also does quite well in explaining the small-scale observations (Zentner \& Bullock
2003).

Our final model is computed in the context of a WDM scenario, in which the primordial power spectrum is scaleinvariant, but free-streaming damps fluctuations below a characteristic scale set by the WDM particle mass $m_{\mathrm{w}}$ :

$$
R_{f}=0.2\left(\Omega_{W D M} \mathrm{~h}^{2}\right)^{1 / 3}\left(\frac{m_{\mathrm{w}}}{\mathrm{keV}}\right)^{-4 / 3} \text { Mpc. }
$$

We calculate the WDM spectra assuming the same flat cosmology with $\Omega_{\mathrm{w}}=\Omega_{m}=0.3$, and use the approximate WDM transfer function given by Bardeen et al. (1986):

$$
P(k)=\exp \left[-k R_{f}-\left(k R_{f}\right)^{2}\right] P_{C D M}(k) .
$$

We choose $m_{\mathrm{w}}=1.5 \mathrm{keV}$, or $R_{f} \simeq 0.027 \mathrm{Mpc}$, in order to suppress power below a mass scale of $\sim 10^{10} M_{\odot}$. This choice of WDM mass (with $n_{s}=1.0$ ) significantly alleviates the usual small scale problems (Eke et al. 2001; Alam et al. 2002; Zentner \& Bullock 2003), yet is not ruled out by Ly- $\alpha$ forest data (Narayanan et al. 2000) or by preWMAP constraints from reionization and early structure formation (Barkana et al. 2001). We neglect the finite particle velocity dispersion, as well as associated phase-space restrictions, which are expected to have negligible consequence for a $1.5 \mathrm{keV}$ model (Alam et al. 2002; Zentner 2003).

\section{HALO COLLAPSE RATES}

We show in Fig. 1 the fraction of the total mass in the Universe that is in collapsed halos in two different mass ranges, computed using the Press-Schechter formalism (Press \& Schechter 1974). In the top panel, we show the fraction of mass in halos with mass greater than $1.0 \times 10^{6} \mathrm{~h}^{-1} M_{\odot}$, but with temperature less than $10^{4} \mathrm{~K}$ (conversions between mass and temperature are as in Somerville \& Primack 1999). As discussed below, we expect gas in these halos (sometimes called "mini-halos") to be able to cool only via $\mathrm{H}_{2}$, and based on the results of hydrodynamic simulations by Yoshida et al. (2003), they may potentially harbor massive Pop III stars. In the bottom panel, we show the mass fraction in halos with $T_{\text {vir }}>10^{4} \mathrm{~K}$, which should be able to cool via $\mathrm{H}_{\mathrm{I}}$ and form Pop II stars with a normal Salpeter-like IMF. We see that all models with reduced small scale power have dramatically decreased mass fractions relative to Standard $\Lambda \mathrm{CDM}$ in halos of the scale expected to be eligible for star formation at high redshift. The TILT model shows the smallest decrease on all scales, while the WDM model shows the most dramatic decrease.

\section{STAR FORMATION AND IONIZING PHOTONS}

A basic requirement for star formation is the presence of cold, dense gas. The two primary coolants in the early Universe, before the production of heavy elements, are molecular and atomic hydrogen. Atomic hydrogen is inefficient at temperatures below $\sim 10^{4} \mathrm{~K}$, implying that molecular hydrogen was probably the main coolant in the first halos to collapse at $z \sim 20-30$, with temperatures of a few hundred Kelvin. Recent theoretical work suggests that the first stars to form in these primordial halos may have been extremely massive, $\sim 100-600 M_{\odot}$, but that the efficiency was rather low, with less than $\sim 1$ percent of the available gas converted to stars or star clusters (Abel et al. 2000, 2002; Bromm et al. 2002). 
The overall efficiency of early star formation is regulated in mini-halos by a complex interplay between the destruction of $\mathrm{H}_{2}$ by UV photons and its catalysis by Xrays (Machacek et al. 2001, 2003; Ricotti et al. 2001, 2002; Cen 2002), and in larger halos by photo-evaporation and supernova feedback (Ciardi et al. 2000; Somerville 2002; Benson et al. 2002). The efficiency of production and mixing of heavy elements also determines the epoch at which the IGM becomes sufficiently polluted with metals to allow cooling to lower temperatures and fragmentation, leading to a shift from the formation of solely supermassive stars to a more normal Salpeter-like IMF (Bromm et al. 2001a). For a more detailed discussion of this scenario, see the excellent review by Loeb \& Barkana (2001), as well as recent papers by Cen (2002), Haiman \& Holder (2003), and Wyithe \& Loeb (2002, 2003).

We model the global star formation rate density (SFRD) by assuming that it is proportional to the rate at which gas collapses into halos in a given mass range:

$$
\dot{\rho}_{*}=e_{*} \rho_{b} \frac{\mathrm{d} F_{h}}{\mathrm{dt}}\left(M>M_{\text {crit }}\right),
$$

where $\frac{\mathrm{d} F_{h}}{\mathrm{dt}}\left(M>M_{\text {crit }}\right)$ is the time derivative of the fraction of the total mass in collapsed halos with masses greater than $M_{\text {crit }}$, obtained from the halo mass function $\frac{\mathrm{d} n_{h}}{\mathrm{~d} M}(M, z)$ given by the Press-Schechter model, and $\rho_{b}$ is the mean density of baryons. We adopt $M_{\text {crit }}=M\left(T_{\text {vir }}=\right.$ $10^{4} \mathrm{~K}$ ) and $e_{*}^{\mathrm{II}}=0.1$ for 'Pop II' halos, and $e_{*}^{\mathrm{III}}=0.002$ and $M_{\text {crit }}^{\mathrm{III}}=1.0 \times 10^{6} \mathrm{~h}^{-1} M_{\odot}$ for 'Pop III' halos. In Somerville \& Livio (2003, SL03), we found that this simple prescription, with these parameter values, agrees well in the redshift range $3 \lesssim z \lesssim 30$ with more detailed semi-analytic models and hydrodynamic simulations of Pop II star formation including photoionization and supernova feedback (e.g. Somerville et al. 2001; Springel \& Hernquist 2003), and also with the general analytic arguments outlined by Hernquist \& Springel (2002). The global star formation rate predicted by this approach is also consistent with observational constraints at $3 \lesssim z \lesssim 6$ (SL03). Similarly, our Pop III SFRD is in good agreement with the detailed numerical hydrodynamic simulations of Pop III formation by Yoshida et al. (2003), as also shown explicitly in SL03. In both cases, the analytic recipe used here slightly overestimates the star formation rate relative to the more realistic simulations, thus leading to more optimistic results for early reionization. As we do not know when the transition from solely supermassive Pop III stars to Pop II star formation will occur, we conservatively shut off the Pop III mode at $z<6$ (when the IGM is known to be significantly polluted with metals). Earlier shut-off times will only lead to later reionization, making our conclusions even stronger.

For Pop II stars, we use the results of Leitherer et al. (1999) for the number of $\lambda<912 \AA$ photons produced by low metallicity stars with a "bottom-light" Salpeter IMF. At ages less than about 3 million years, these stars produce about $d n_{\gamma} / d t=8.9 \times 10^{46}$ photons s ${ }^{-1} M_{\odot}^{-1}$. For Pop III, we assume that each star produces $1.6 \times 10^{48}$ photons $\mathrm{s}^{-1} M_{\odot}^{-1}$ for a lifetime of 3 million years (Bromm et al. 2001b). We note that, in the spirit of optimism that we have adopted throughout, these values are at the high end of the estimates of ionizing photon production and lifetime for both populations.

The cumulative number of ionizing photons per hydrogen atom in the Universe is shown in Fig. 2, for each of the four models summarized in Table 1 . The ionized fraction $x_{e}$ is expected to scale as this quantity times $f_{\text {esc }} f_{\text {ion }} / C_{\text {clump }}$ (e.g., Spergel et al. 2003), where $f_{\text {ion }}$ is the number of ionizations per UV photon, $f_{\mathrm{esc}}$ is the fraction of ionizing photons that escape from the galaxy, and $C_{\text {clump }}$ is the clumping factor, reflecting the clumpiness of the IGM. Many analytic and numerical studies have addressed the issue of the precise number of ionizing photons per atom needed to attain 'overlap', or reionization that is $\sim 99$ percent complete (Sokasian et al. 2003; Ciardi et al. 2003b; Razoumov et al. 2002; Haiman et al. 2001; Miralda-Escudé et al. 2000; Gnedin 2000; Madau et al. 1999). This number is a function of the local IGM density (thus, indirectly, of the redshift of reionization), and of the masses of the halos harboring the sources, as more massive sources are expected to be in denser environments with higher clumping factors. For reference, Sokasian et al. (2003) find (in the highest resolution numerical simulation of reionization by stellar sources to date) that a gross budget (i.e., before accounting for the escape fraction) of about 5-20 photons per $\mathrm{H}$ atom was required to achieve a volume-weighted ionization fraction of $99 \%$. The clumping factors in numerical simulations are known to be potentially overestimated due to the limited numerical resolution (Haiman et al. 2001; Sokasian et al. 2003), and no numerical simulation to date has included the contribution from Pop III stars, which are likely to be less clustered. We therefore entertain the possibility that as few as $\sim 2$ ionizing photons per $\mathrm{H}$ atom may be able to do the job. The redshift at which a total of 2 or 10 ionizing photons per $\mathrm{H}$ atom have been produced in each of the models is shown in Fig. 2, and recorded in Table 1. We regard these values as bracketing a generous but plausible range for the expected redshift of overlap in these models.

One can quickly see that it is difficult to achieve reionization earlier than $z \sim 10$ in any of the models with reduced small scale power. In the RSI model favored by Spergel et al. (2003), the number of ionizing photons per hydrogen atom $n_{\gamma}$ reaches the most optimistic range for expected overlap $\left(n_{\gamma} \gtrsim 2\right)$ at $z \sim 8$ - only slightly earlier than the redshift at which the WDM model reaches this range $(z \sim 7)$. Values of $n_{\gamma} \gtrsim 10-20$, corresponding to more typically favored values of the photon escape fraction $\left(f_{\text {esc }} \sim 0.2\right)$, recombination rate, and clumping factor, are attained only at $z \sim 5.8-4.8$ in the models with reduced small scale power - thus these models (particularly WDM and RSI) may not even be able to reionize the Universe early enough for consistency with the $S D S S$ quasar observations. As noted above, we have allowed massive Pop III star formation to continue until $z=6$, although we actually expect it to shut off much earlier. This would lead to even later reionization, as shown by the short-dashed lines in Fig. 2, which show the contribution from Pop II stars only.

\subsection{Extreme Parameter Values}

Certainly it may be argued that the IMF and formation efficiency of early generations of stars are highly uncertain. We now consider how much we would have to vary the free 
parameters in our simple model to obtain reionization by $z \sim 17$ in the RSI model favored by the analysis of WMAP combined with other data (Spergel et al. 2003). These results are summarized in Table 2. In model RSI.x1, we increase the efficiency of star formation in 'Pop II' halos to $100 \%$ (corresponding to all baryons forming stars the moment they are within a halo) and the assumed mass of Pop III stars to $600 M_{\odot}$, the maximum expected value suggested by Omukai \& Palla (2003), though this is larger than the maximum value advocated by Abel et al. (2002). Reionization is then expected between $z \sim 10.5-8$. Only if we assume $100 \%$ star formation efficiency in Pop III halos as well (model RSI.x2) do we find that reionization could plausibly occur by $z \sim 16-14$, in reasonable agreement with the WMAP TE results.

Alternatively, we can leave the star formation efficiency parameters at their fiducial values and vary the number of ionizing photons produced by each population. For example, the IMF of early Pop II stars might be topheavy, because of the higher temperatures and pressures and lower metallicities at early times (Larson 1998). Moreover, Tumlinson et al. (2003) find that zero-metallicity Pop II stars with a Salpeter IMF produce only about $50 \%$ more hydrogen-ionizing photons than the low-metallicity Leitherer et al. (1999) models used here (see also Schaerer 2003). We find that increasing the number of ionizing photons per solar mass of Pop II stars by a factor of 2-10 can only shift reionization to at best $z_{\text {reion }} \sim 10$ (models RSI.x3 and RSI.x4). Even with the extreme assumption that Pop II stars produce as many ionizing photons per unit mass as supermassive Pop III stars (about 20 times the fiducial value), we find it is unlikely that the Universe could be reionized earlier than $z \sim 11$ in the context of the WMAP RSI model.

\section{DISCUSSION AND CONCLUSIONS}

We have investigated early structure formation in the Standard $\Lambda$ CDM model and in three models with reduced small scale power, each of which alleviates conflicts with observations on sub-galactic scales at low redshift. All the models we considered are consistent with the WMAP data on large scales, and two of them (TILT and RSI) are favored by the combined analysis of WMAP and other CMB data with Lyman- $\alpha$ forest and 2dFGRS data (Spergel et al. 2003). We model star formation using a simple recipe that has been calibrated against more detailed semi-analytic and numerical hydrodynamic simulations of Pop II and massive Pop III star formation (Somerville \& Livio 2003; Springel \& Hernquist 2003; Yoshida et al. 2003). Using these fiducial parameter values, and assuming that a gross production of between 2-10 ionizing photons per $\mathrm{H}$ atom is needed to attain overlap, we estimate that the Universe would become reionized between $z \sim 13$ and $z \sim 9$ with the Standard $\Lambda$ CDM power spectrum, in agreement with several other semi-analytic and numerical studies of reionization (Gnedin 2000; Razoumov et al. 2002; Haiman \& Holder 2003; Ciardi et al. 2003a; Sokasian et al. 2003). Using the same approach, we find that none of the models with reduced small scale power can produce at least two ionizing photons per atom before $z \sim 9.5$, which is about $1.5 \sigma$ lower than the epoch of reionization favored by Kogut et al. (2003) based on the WMAP TE measurement. More plausible values of $n_{\gamma} \sim 10$ are not attained until $z \lesssim 6$ in the models with reduced power on small scales. The WDM and RSI models may have difficulty reionizing the Universe even by $z \gtrsim 6$, as required by observations of high redshift quasars (Fan et al. 2001). Of the three models we considered, the model with a fixed tilt $n_{s}=0.95$ (TILT) is the best candidate for obtaining a compromise between the requirements of early structure formation and observed galaxy central densities and sub-structure at low redshift. The RSI model favored by Spergel et al. (2003) is only marginally better than WDM in terms of the reionization constraints. It is also worth noting that any attempts to push reionization to higher redshift by increasing the small scale power (e.g., by adopting a 'red tilt' $n>1$ as suggested by Cen 2003) would further exacerbate the problems on sub-galactic scales.

We find that varying the efficiency of star formation or the stellar IMF within a reasonable range of values cannot significantly change these conclusions. Only if the efficiency of star formation is pushed to an extreme upper limit $\left(100 \%\right.$ of baryons in halos with $M>1 \times 10^{6} \mathrm{~h}^{-1} M_{\odot}$ turn instantly into stars) can the RSI model plausibly reionize the Universe by $z \sim 16-14$. This result is in agreement with a similar analysis performed by Haiman \& Holder (2003). If such extreme star formation were allowed to continue to lower redshift (even to $z \sim 4$ ), such a model would be in clear conflict with observations of galaxies and the IGM. Even stronger constraints will soon be obtained from direct observations of $z>6$ galaxies with HST and the planned JWST telescope.

We conclude that if we require that the Universe was reionized at least by $z \sim 12$, within $\sim 1 \sigma$ of the WMAP result, then, in the context of the current framework, this paradox can be resolved only by adopting one or more of the following: (1) the efficiency of Pop III star formation is much higher than current theory suggests, (2) there is an additional population of ionizing sources (such as miniquasars) at high redshift, (3) the power spectrum has some higher-order feature that produces an upturn in power at masses just below the scale of dwarf galaxies, or (4) we must retain the scale-invariant, $n=1$ standard $\Lambda \mathrm{CDM}$ power spectrum, and somehow solve the small-scale problems in another way. While none of these solutions is particularly attractive, we tend to favor number (4) from the context of reionization, although this has its own difficulties. If reionization indeed occurred early, and the small-scale problems are as robust as they appear, then there are significant gaps in our theoretical understanding of first light and the structure of galaxies on $\mathrm{kpc}$ scales. The connection between the two certainly warrants more examination.

\section{ACKNOWLEDGMENTS}

We acknowledge Lars Hernquist, Joel Primack, Naoki Yoshida, and Andrew Zentner for useful discussions. JSB would like to thank Rosemary Wyse for generous hospitality during his visit to Johns Hokins University, where a substantial fraction of this work was completed. JSB is provided for by NASA through Hubble Fellowship grant HF-01146.01-A from the Space Telescope Science Institute, which is operated by the Association of Universities for Research in Astronomy, Incorporated, under NASA contract NAS5-26555. 


\section{REFERENCES}

Abel, T., Bryan, G. L., \& Norman, M. L. 2000, ApJ, 540, 39

-. 2002, Science, 295, 93

Alam, S. M. K., Bullock, J. S., \& Weinberg, D. H. 2002, ApJ, 572, 34

Avila-Reese, V., Colín, P., Valenzuela, O., D’Onghia, E., \& Firmani, C. 2001, ApJ, 559, 516

Bardeen, J. M., Bond, J. R., Kaiser, N., \& Szalay, A. S. 1986, ApJ, 304,15

Barkana, R., Haiman, Z., \& Ostriker, J. P. 2001, ApJ, 558, 482

Becker, R. H. et al. 2001, AJ, 122, 2850

Benson, A. J., Lacey, C. G., Baugh, C. M., Cole, S., \& Frenk, C. S. 2002, MNRAS, 333, 156

Bode, P., Ostriker, J. P., \& Turok, N. 2001, ApJ, 556, 93

Bromm, V., Coppi, P. S., \& Larson, R. B. 2002, ApJ, 564, 23

Bromm, V., Ferrara, A., Coppi, P. S., \& Larson, R. B. 2001a, MNRAS, 328, 969

Bromm, V., Kudritzki, R. P., \& Loeb, A. 2001b, ApJ, 552, 464

Bullock, J. \& Zentner, A. 2002, in Dark Matter 2002, astro$\mathrm{ph} / 0207534$

Cen, R. 2002, preprint, astro-ph/0210473

-. 2003, preprint, astro-ph/0303236

Ciardi, B., Ferrara, A., Governato, F., \& Jenkins, A. 2000, MNRAS, 314,611

Ciardi, B., Ferrara, A., \& White, S. D. M. 2003a, preprint, astro$\mathrm{ph} / 0302451$

Ciardi, B., Stoehr, F. \& White, S. 2003b, preprint, astro-ph/0301293

Colín, P. Avila-Reese, V., \& Valenzuela, O. 2000, ApJ, 542, 622

Eke, V. R., Navarro, J. F., \& Steinmetz, M. 2001, ApJ, 554, 114

Fan, X. et al. 2001, AJ, 122, 2833

Flores, R. A. \& Primack, J. R. 1994, ApJ, 427, L1

Gnedin, N. Y. 2000, ApJ, 535, 530

Haiman, Z., Abel, T., \& Madau, P. 2001, ApJ, 551, 599

Haiman, Z. \& Holder, G. 2003, preprint, astro-ph/0302403

Hannestad, S., Hansen, S. H., Villante, F. L., \& Hamilton, A. J. S. 2002, Astroparticle Physics, 17, 375

Hernquist, L. \& Springel, V. 2002, preprint, astro-ph/0209183

Hui, L. \& Haiman, Z. 2003, preprint, astro-ph/0302439

Kinney, W. H. 2003, astro-ph/0301448

Klypin, A., Kravtsov, A. V., Valenzuela, O., \& Prada, F. 1999, ApJ, 522,82

Knebe, A., Devriendt, J. E. G., Mahmood, A., \& Silk, J. 2002, MNRAS, 329, 813

Kogut, A. et al. 2003, preprint, astro-ph/0302213

Kosowsky, A. \& Turner, M. S. 1995, Phys. Rev. D, 52, 1739
Larson, R. B. 1998, MNRAS, 301, 569

Leitherer, C. et al. 1999, ApJS, 123, 3

Loeb, A. \& Barkana, R. 2001, ARA\&A, 39, 19

Machacek, M. E., Bryan, G. L., \& Abel, T. 2001, ApJ, 548, 509

- 2003, MNRAS, 338, 273

Madau, P., Haardt, F., \& Rees, M. J. 1999, ApJ, 514, 648

McGaugh, S. S., Barker, M. K., \& de Blok, W. J. G. 2003, ApJ, 584, 566

Miralda-Escudé, J., Haehnelt, M., \& Rees, M. J. 2000, ApJ, 530, 1

Moore, B. 1994, Nature, 370, 629

Moore, B., Ghigna, S., Governato, F., Lake, G., Quinn, T., Stadel, J., \& Tozzi, P. 1999, ApJ, 524, L19

Narayanan, V. K., Spergel, D. N., Davé, R., \& Ma, C. 2000, ApJ, 543, L103

Omukai, K. \& Palla, F. 2003, preprint, astro-ph/0302345

Press, W. H. \& Schechter, P. 1974, ApJ, 187, 425

Razoumov, A. O., Norman, M. L., Abel, T., \& Scott, D. 2002, ApJ, 572,695

Ricotti, M., Gnedin, N. Y., \& Shull, J. M. 2001, ApJ, 560, 580

-. 2002, ApJ, 575, 49

Schaerer, D. 2003, A\&A, 397, 527

Schirber, M. \& Bullock, J. S. 2003, ApJ, 584, 110

Sokasian, A., Abel, T., Hernquist, L., \& Springel, V. 2003, preprint, astro-ph/0303098

Somerville, R. \& Livio, M. 2003, ApJ, accepted, astro-ph/0303017

Somerville, R. S. 2002, ApJ, 572, L23

Somerville, R. S. \& Primack, J. R. 1999, MNRAS, 310, 1087

Somerville, R. S., Primack, J. R., \& Faber, S. M. 2001, MNRAS, 320,504

Sommer-Larsen, J. \& Dolgov, A. 2001, ApJ, 551, 608

Spergel, D. N. et al. 2003, preprint, astro-ph/0302209

Springel, V. \& Hernquist, L. 2003, MNRAS, 339, 312

Swaters, R. A., Madore, B. F., van den Bosch, F. C., \& Balcells, M. 2003, ApJ, 583, 732

Tumlinson, J., Shull, J. M., \& Venkatesan, A. 2003, ApJ, 584, 608

van den Bosch, F. C., Mo, H. J., \& Yang, X. 2003, preprint, astro$\mathrm{ph} / 0210495$

Wyithe, S. \& Loeb, A. 2002, preprint, astro-ph/0209056

-. 2003, preprint, astro-ph/0302297

Yoshida, N., Abel, T., Hernquist, L., \& Sugiyama, N. 2003, preprint, astro-ph/0301645

Zentner, A. R. 2003, Ph.D. Thesis

Zentner, A. R. \& Bullock, J. S. 2002, Phys. Rev. D, 66, 43003

-. 2003, in preparation 
TABLE 1

SUMMARY OF MODELS

\begin{tabular}{lcccccc}
\hline Model & $n\left(k_{W M A P}\right)$ & $\mathrm{d} n / \mathrm{d} \ln k$ & $\sigma_{8}$ & $m_{\nu}(\mathrm{keV})$ & $z\left(n_{\gamma}=2\right)$ & $z\left(n_{\gamma}=10\right)$ \\
\hline$\Lambda$ CDM & 1.0 & 0.0 & 0.90 & 0.0 & 13.3 & 8.7 \\
TILT & 0.95 & 0.0 & 0.75 & 0.0 & 9.4 & 5.8 \\
RSI & 0.93 & -0.03 & 0.81 & 0.0 & 7.9 & 5.0 \\
WDM & 1.0 & 0.0 & 0.89 & 1.5 & 6.9 & 4.8 \\
\hline
\end{tabular}

TABLE 2

RSI MODEL VARIANTS

\begin{tabular}{ccccccc}
\hline model & $e_{*}^{\mathrm{III}}$ & $e_{*}^{\mathrm{III}}$ & $N_{\gamma}^{\mathrm{II}}$ & $z\left(n_{\gamma}=1\right)$ & $z\left(n_{\gamma}=2\right)$ & $z\left(n_{\gamma}=10\right)$ \\
\hline RSI.x1 & 1.0 & 0.006 & $\times 1$ & 11.4 & 10.5 & 8.6 \\
RSI.x2 & 1.0 & 1.0 & $\times 1$ & 16.5 & 15.8 & 13.9 \\
RSI.x3 & 0.1 & 0.002 & $\times 2$ & 9.6 & 8.7 & 6.0 \\
RSI.x4 & 0.1 & 0.002 & $\times 10$ & 11.14 & 10.3 & 8.4 \\
RSI.x5 & 0.1 & 0.002 & $N_{\gamma}^{\mathrm{III}}$ & 11.7 & 11.0 & 9.1 \\
\hline
\end{tabular}




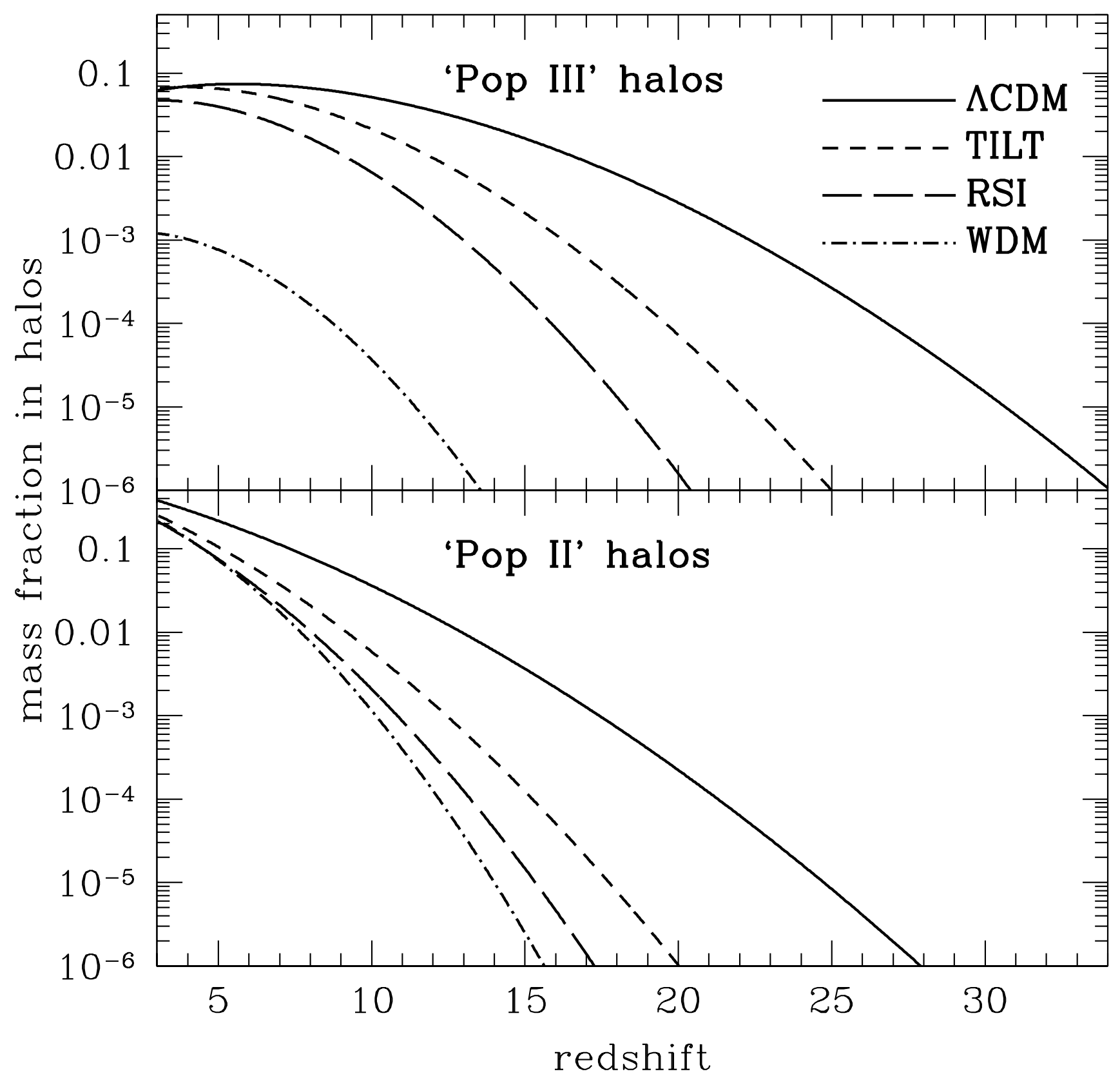

FIG. 1. - The fraction of mass in collapsed dark matter halos as a function of redshift for the four power spectra summarized in Table 1. The top panel shows the mass fraction in halos with mass greater than $1.0 \times 10^{6} \mathrm{~h}^{-1} M_{\odot}$ but virial temperature $T_{\text {vir }}<10^{4}$ $\mathrm{K}$. In the absence of metals, gas in these halos can cool primarily by molecular hydrogen, and we associate them with possible sites of massive $\left(200 M_{\odot}\right)$ Pop III star formation. The bottom panel shows the mass fraction in halos with $T_{\text {vir }}>10^{4} \mathrm{~K}$. We associate these halos with $\mathrm{H}_{I}$ cooling and Pop II star formation with a 'normal' IMF. The impact of reduced small scale power on early star formation on both of these mass scales is dramatic. 


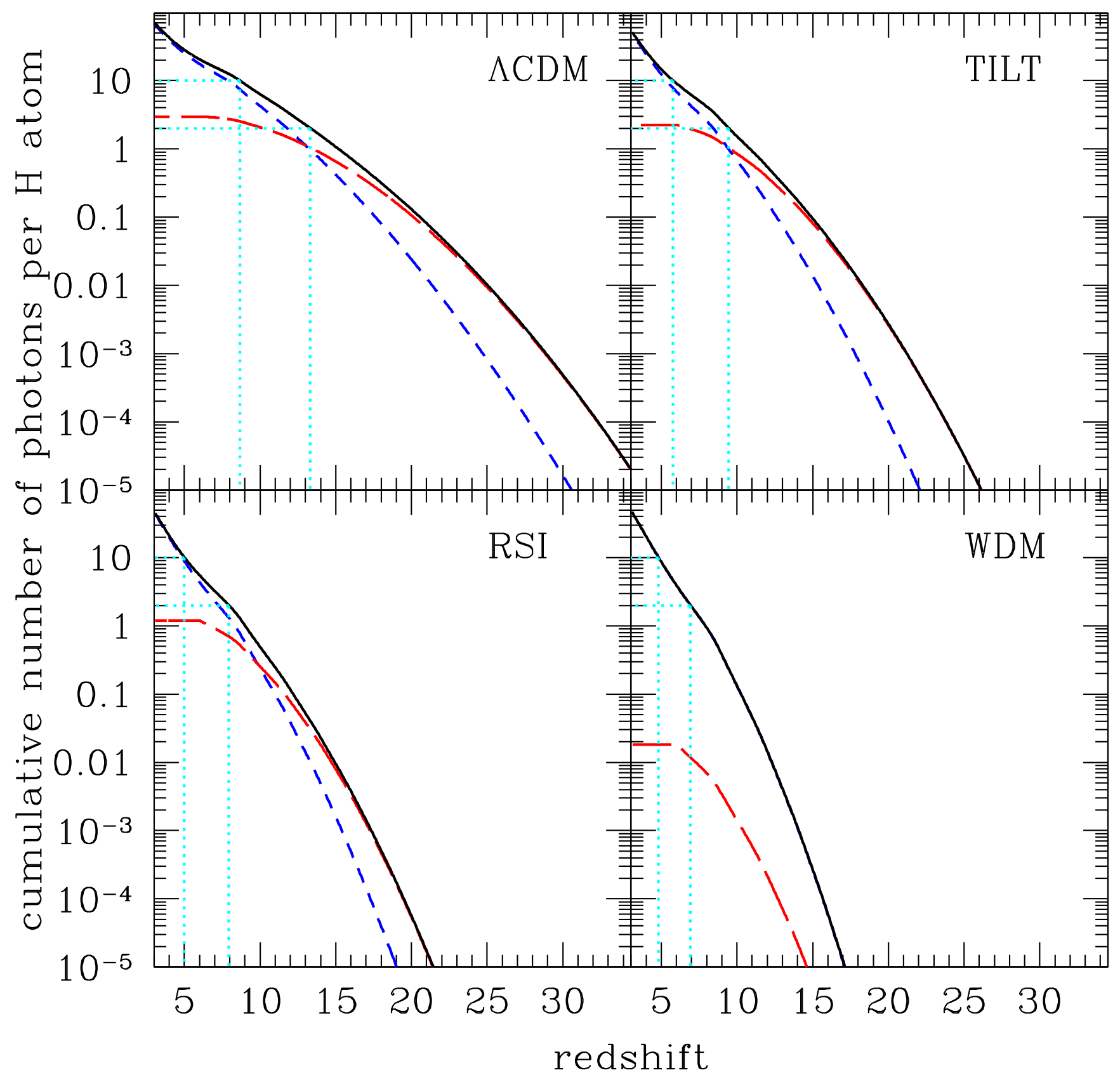

FIg. 2.- The cumulative number of hydrogen-ionizing photons per hydrogen atom produced, as a function of redshift, for the four initial power spectra. Long dashed lines show the contribution from Pop III stars, short dashed lines show the contribution from Pop II stars, and solid lines show the total. Straight dotted lines indicate the redshift at which 2 and 10 ionizing photons per atom have been produced, and roughly bracket the range of redshifts when the overlap of ionized regions is expected to occur. In all three models with reduced small scale power, overlap occurs later than $z \sim 10$. This may be compared with the estimate of $z_{\text {reion }}=17 \pm 5$ from the $W M A P$ temperature-polarization results (Kogut et al. 2003). 